\title{
T-ray multilayer interference filter
}

\author{
W. Withayachumnankul, B. M. Fischer, S. P. Mickan, and D. Abbott \\ School of Electrical \& Electronic Engineering, The University of Adelaide, SA 5005, Australia \\ Email: withawat@eleceng.adelaide.edu.au
}

\begin{abstract}
An optical multilayer interference filter is made from two or more different dielectric materials layered in such a way that it promotes constructive/destructive wave interference for a selected frequency in the direction normal to the layers. Usually, each layer has the thickness of a quarter of wavelength at which the stopband is required. In this paper, a quarter-wave interference filter is realised for T-ray applications. The dielectric materials used are high-resistivity silicon and free space, both of which have high transparency to T-rays and flat responses over the frequencies of interest. The designed thickness of both materials is in the order of a hundred microns. The THz-TDS measurement of the finished structure is in agreement with theory.
\end{abstract}

\section{INTRODUCTION}

A number of T-ray filters have been realized to date, for example, photonic bandgap crystals [1], perforated metal sheets [2], and multilayer interference filters. A multilayer interference filter is an attractive option because of its unsophisticated structure. Using alternating thin films of T-ray transparent materials, with a proper index arrangement, full control over a particular frequency band is attainable via the interference mechanism. A similar multilayer structure is exploited for a T-ray mirror [3].

This paper introduces transmission interference filters covering the frequency gap of ultrafast T-ray systems, i.e. between 0.1 and $1.0 \mathrm{THz}$, where requirements of these filters are foreseeable.

\section{QUARTER-WAVE MULTILAYER INTERFERENCE FILTER}

Fig. 1 illustrates a multilayer interference filter, one period of which is composed of two stratified layers made from materials with different indices of refraction. Typically, the optical thickness of each individual layer is a quarter of the central wavelength, $\lambda_{c}$, of the fundamental stopband [4]. This condition is formulated as

$$
n_{H} l_{H}=n_{L} l_{L}=\frac{\lambda_{c}}{4}=\frac{c}{4 f_{c}},
$$

where the subscriptions $H$ and $L$ are for high- and low-indexed materials, $n$ for the refractive index, and $l$ for the thicknesses.

An explanation for the above equation is as follows: a slab of any material produces Fabry-Pérot fringes, the minima of which can be utilized for stopbands. However, a single slab cannot cause wide and deep frequency gaps. Thus, cascading of a number of slabs made from two or more different materials is required. In order to arrange the frequency gaps efficiently, the fringes' maxima and minima of cascaded slabs must be at the same spectral position to enhance constructive and destructive interference at particular frequencies. This is possible through optical length equalization, as used in Equation 1.

\section{FILTER REALIZATION}

As mentioned previously, the stopband and passband are governed by an interference mechanism through a multilayer material structure. The high efficiency of interference mechanism is achievable, given that each material is non-dispersive and non-absorptive in the T-ray frequency band.

Two candidate materials, which reasonably satisfy the above requirements and have a large difference in refractive index, are selected for this experiment. The first material, assigned $H$, is high-resistivity silicon, in particular float-zone (FZ) silicon. Undoped FZ silicon is known to have negligible absorption and a constant refractive index of 3.418 at $\mathrm{T}$ ray frequencies [5]. The second material, assigned $L$, is free space which assumably has the unity refractive index and zero absorption over the electromagnetic spectrum.

FZ silicon wafers supplied by Siltronix have a thickness of $50 \pm 5 \mu \mathrm{m}$. Due to the Fabry-Pérot effect, or particularly the destructive interference, the transmission of a single wafer has a minimum at $f_{c}=c /\left(4 n_{\mathrm{si}} l_{\mathrm{si}}\right)=0.439 \mathrm{THz}$. This frequency is then set as the central frequency of the fundamental stopband. Accordingly, the thickness of an air gap, $l_{L}=170.9 \mu \mathrm{m}$, is chosen to have minimum transmission at this frequency.

\section{RESUlTS}

A series of silicon-air filters with various periods are characterised by transmission THz-TDS, with a spectrometer having a bandwidth from 0.1 to $1.0 \mathrm{THz}$ and the maximum dynamic range of $25 \mathrm{~dB}$. The measurement for each filter is carried out nine times, and the time-domain pulses are averaged. For each recorded signal the time window is $555.22 \mathrm{ps}$ with the sampling interval of 33.9 fs. Fourier transform of the pulse yields the spectral resolution of $1.8 \mathrm{GHz}$.

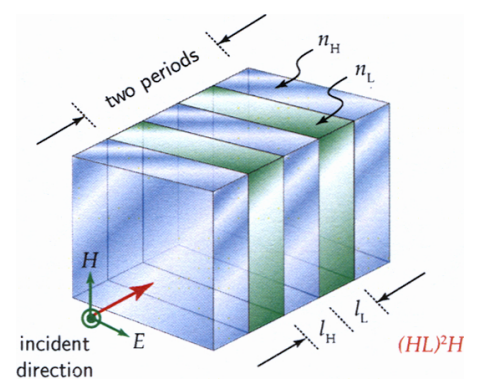

Fig. 1. Multilayer interference filter. The structure comprises a number of dielectric periods. Each period is constructed from high- and low-indexed materials with the indices of refraction of $n_{H}$ and $n_{L}$, and the thicknesses of $l_{H}$ and $l_{L}$, respectively. The figure shows a $H L H L H$ or $(H L)^{2} H$ structure, where $H$ and $L$ represent high- and low-indexed materials, respectively. 


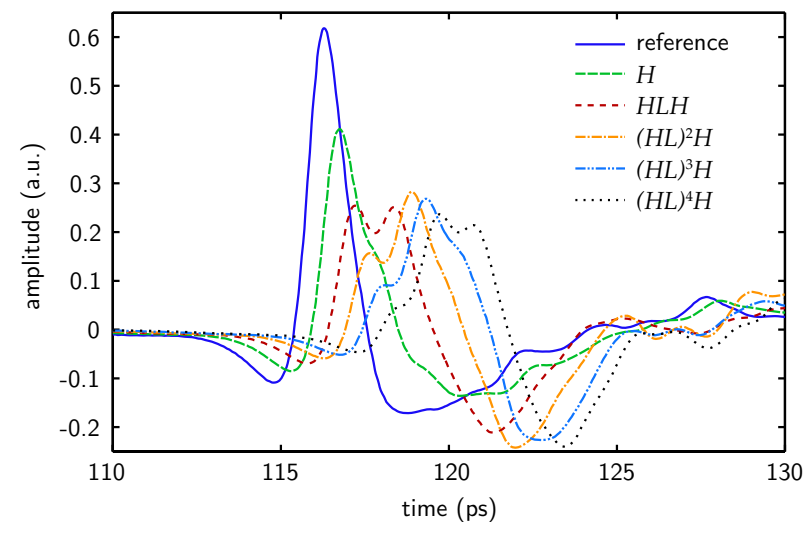

Fig. 2. T-ray signals transmitted through the interference filters. The $H$ measurement actually represents the signal recorded from a single 50-micron silicon wafer. The pulses are delayed and reshaped by the filters.

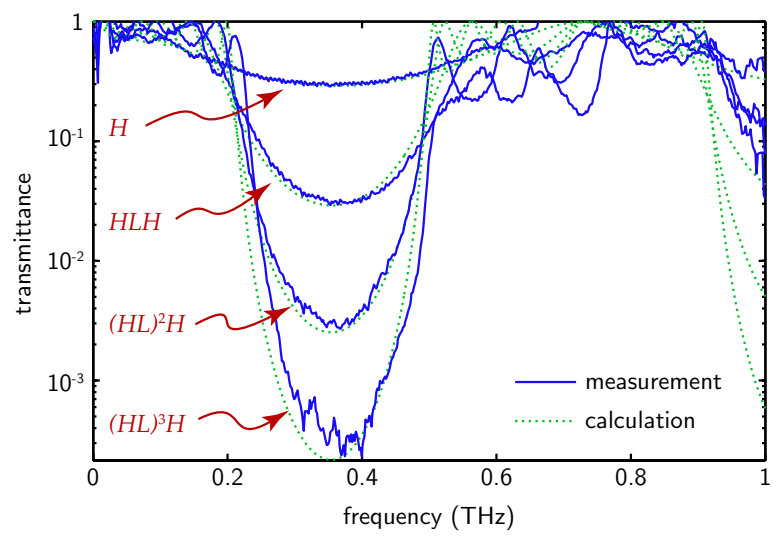

Fig. 3. Transmittance of the interference filters. For each filter, the first stopband resides between 0.2 and $0.5 \mathrm{THz}$, centered at $0.36 \mathrm{THz}$. The successive stopbands at higher frequencies are unresolvable due to the system bandwidth limit. The spectrum inside the stopband of the $(H L)^{3} H$ filter is noisy, because the attenuation reaches the noise floor.

Fig. 2 shows a set of pulses recorded from the designed interference filters. Notice that the pulses for $(H L)^{n} H$ structures do not exhibit much difference in the maximum amplitude, but rather their shapes. This is because the filters attenuate the amplitude at the specific frequency range and preserve the rest.

The transmittances in Fig. 3 are calculated from the spectra of the reference, $E_{\text {ref }}(\omega)$, and of the filter, $E_{\text {fil }}(\omega)$, using $T(\omega)=\left|E_{\mathrm{fil}}(\omega) / E_{\mathrm{ref}}(\omega)\right|^{2}$. In the figure, the center of the first stopband for every structure is at approximately $0.36 \mathrm{THz}$, while the width of the stopband is $\approx 0.2 \mathrm{THz}$. As the period of the filters increases, the attenuation in the stopband increases logarithmically, roughly at an order of magnitude per period. Despite the strong attenuation in the stopband, the filters have relatively small attenuation in the passband, i.e. between 0.0 and $0.2 \mathrm{THz}$, and between 0.5 to $0.9 \mathrm{THz}$. Unfortunately, the system bandwidth is not enough to render the second stopband, which is beyond $0.9 \mathrm{THz}$. In complementary to transmittance, the phase response extracted using $\phi(\omega)=\angle E_{\text {fil }}(\omega)-\angle E_{\text {ref }}(\omega)$ is shown in Fig. 4. It would be obvious that the phase is anomalous in the stopband, in between 0.2 to $0.5 \mathrm{THz}$.

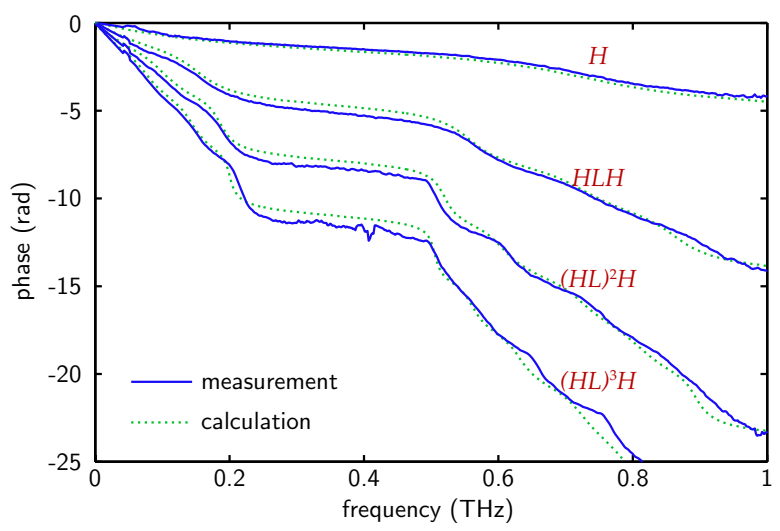

Fig. 4. Phase response of the interference filters. The phases are anomalous inside the stopband.

Despite the appearance of the stopband, the central frequency of the stopband deviates from the design at $0.439 \mathrm{THz}$. This would be more likely from uneven surfaces of the air-gap rings, which result in thicker air layers. Hence, in the simulation, an adjustment to the material parameters is performed in compensation to the structural uncertainties. Through the fitting using a characteristic matrix analysis [6], the thicknesses of the silicon wafers and air gaps are changed to $60 \mu \mathrm{m}$ and $220 \mu \mathrm{m}$, respectively. The simulation results accompany the measured transmittances and phase responses in Fig. 3 and 4.

\section{CONClusion}

In this paper, multilayer interference filters are designed to be operated in the T-ray frequency regime. The filters are made from several ultrathin silicon wafer and air gap layers. The shape, width, and position of a stopband are controlled through a combination of the material thicknesses, refractive indices, and a number of periods. Characterised by THz-TDS, the filters show the stopband spanning 0.2 to $0.5 \mathrm{THz}$ with the central frequency at $0.36 \mathrm{THz}$. The transmittance in the stopband decreases at approximately an order of magnitude per silicon-air period. A characteristic matrix analysis shows an agreement between the model and the measurement at the estimated thicknesses of the silicon and air gap of $60 \mu \mathrm{m}$ and $220 \mu \mathrm{m}$, respectively.

\section{REFERENCES}

[1] E. Özbay, E. Michel, G. Tuttle, R. Biswas, K. M. Ho, J. Bostak, and D. M. Bloom, "Terahertz spectroscopy of three-dimensional photonic band-gap crystals," Optic. Lett., vol. 19, no. 15, pp. 1155-1157, 1994.

[2] C. Winnewisser, F. T. Lewen, M. Schall, M. Walther, and H. Helm, "Characterization and application of dichroic filters in the 0.1-3 THz region," IEEE Trans. Microw. Theor. Tech., vol. 48, no. 4, pp. 744-749, 2000.

[3] N. Krumbholz, K. Gerlach, F. Rutz, M. Koch, R. Piesiewicz, T. Kürner, and D. Mittleman, "Omnidirectional terahertz mirrors: A key element for future terahertz communication systems," Appl. Phys. Lett., vol. 88, art. no. 202905, 2006.

[4] S. J. Orfanidis, Electromagnetic Waves and Antennas, 2006.

[5] D. Grischkowsky, S. Keiding, M. van Exter, and C. Fattinger, "Farinfrared time-domain spectroscopy with terahertz beams of dielectrics and semiconductors," J. Opt. Soc. Am. B, vol. 7, no. 10, pp. 2006-2015, 1990.

[6] E. Hecht, Optics, 2nd ed. Addison-Wesley, 1987. 